\title{
Osteosarcoma of the Skull: An Unusual Presentation in the Left Parietal-Occipital Region
}

\author{
Aditya Adhav ${ }^{1}$, Sagar Bhalerao ${ }^{1}$, Rajnish Nagarkar', Sirshendu Roy', Samadhan Pawar ${ }^{1}$ \\ ${ }^{1}$ HCG Manavata Cancer Centre, Nashik, Maharashtra.
}

\section{Abstract}

Osteosarcoma (OS) is an uncommon primary malignant brain tumor. The incidence of osteosarcoma of the skull is low with an estimated 3.4 cases per million reported per year. We report a case of OS of the skull in an 18-year-old female patient. She had complained of swelling on the left side of the head accompanied with frequent headache and diminished vision in the left eye. An PET-CT of the skull revealed a large $93 \times 90 \mathrm{~mm}$ lesion in the left parietal-occipital region. Osteoblastic osteogenic sarcoma of the skull was confirmed histopathologically. The patient received six cycles of Adriamycin and cisplatin; is under close observation and currently doing well. It is empirical to report case reports, specifically unusual cases like OS of the skull. Case reports not only help disseminate knowledge but also help streamline diagnostic and treatment approaches for unusual cases.

Keywords: Jugular veins, neck, omohyoid, fenestration.

Corresponding Author: Dr. Aditya Adhav, Consultant, Department of surgical oncology, HCG Manavata Cancer Centre, Off Nashik Expressway, Behind Shivang Auto, Nashik- 422 011, India.

Received: January 2019

Accepted: January 2019

\section{Introduction}

Osteosarcoma (OS) is reasonably rare primary malignant bone tumor. The estimated worldwide incidence is 3.4 cases per million people per year. The five-year survival rates of OS has been extremely low, i.e. $20 \%$ for most of the twentieth century. ${ }^{[1]}$ However, with the introduction of the adjuvant chemotherapy, the survival rates have increased to $50 \% .{ }^{[1]}$ OS accounts for nearly $20 \%$ of all primary bone cancers. ${ }^{[2]}$ An estimated $5-10 \%$ of these cases are located within the craniofacial bones. ${ }^{[2]}$ OS is characterized by the presence of malignant mesenchymal cells that produce immature or osteoid bone. It accounts for roughly $30-80 \%$ of primary skeletal sarcomas. OS is primarily found in children, teenagers, and young adults with the age range of $10-30$ years. ${ }^{[3]}$ We report a case of OS arising in the parietaltemporal bone in a young adult female.

\section{Case Report}

An 18-year-old female patient presents to our hospital with swelling on the left side of the head since two years. The patient complained of frequent headache and diminished vision in the left side. A Positron emission tomographycomputed tomography (PET-CT) of the skull revealed a large $93 \times 90 \mathrm{~mm}$ lesion in the left parietal-occipital region. The lesion involved the bone and extended intra-cranially [Figure 1]. A craniotomy wide-excision of the mass was performed. A histopathological investigation confirmed osteoblastic osteogenic sarcoma of the skull. Postcraniotomy, oozing from the operative site was observed. A left parietal-occipital craniotomy defect was observed. The scalp defect was closed by transpositional flap. A leftparieto-occipital craniotomy defect was observed. The previously visualized left subdural collection was resolved. Mild external herniation of the brain through the craniotomy defect with dilation of the posterior horn of the left lateral ventricle $(30 \mathrm{~mm}$ diameter) was observed. The patient received six cycles of Adriamycin and cisplatin. The patient is currently doing well and under close observation.

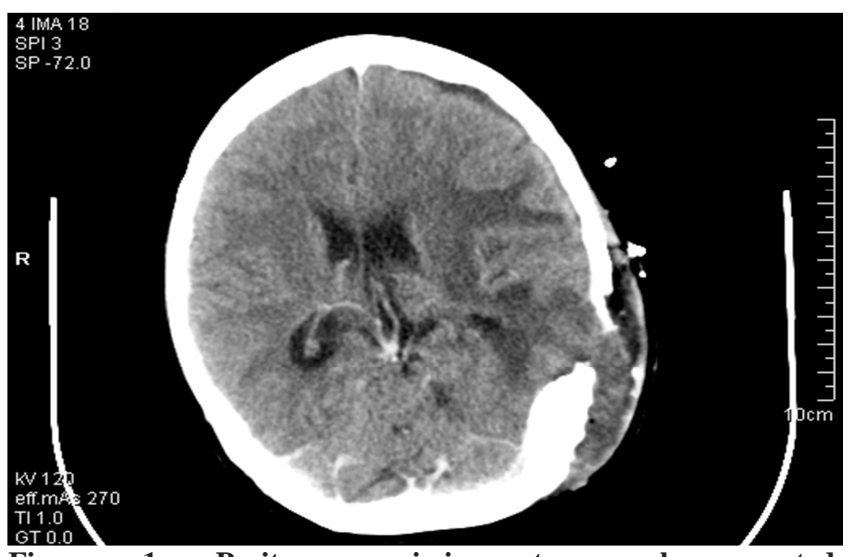

Figure 1: Positron emission tomography-computed tomography (PET-CT) of the skull revealed a large 93x90 mm lesion in the left parietal-occipital region. The lesion involved the bone and extended intra-cranially. 


\section{Discussion}

Bone tumors are uncommon in children with an estimated incidence of 8.7 per million of less than 20 years. ${ }^{[3]}$ The peak incidence of OS is generally observed in the second decade of life, specifically during the adolescent stage as observed in our case. As per current evidence, the incidence of childhood cancer ranges from 75 to 150 per million children worldwide $^{[4]}$ The incidence of childhood cancer in India is 38 to 124 million per children per year. ${ }^{[3,4]}$

Primary osteosarcoma of the skull is an uncommon presentation. The reported incidence is nearly $1 \%$ to $2 \%$ of all skull tumors. To the best of our knowledge, only 150 such cases have been reported in the literature. ${ }^{[5]}$ The etiology of osteosarcoma is unclear while the major risk of development is similar to those of osteosarcoma of the long skeletal bones. ${ }^{[5]}$ The clinical symptoms of primary osteosarcoma of the skull differ and are specific to the tumor site. ${ }^{[6]}$ Patients with osteosarcoma often complaint of frequent headaches, visual impairment, cranial nerve palsies, or cranial hypertension. ${ }^{[6,7]}$ In our case, the patient reported to have headache and diminished left eye vision. Osteosarcomas are spindle-cell tumors that are associated with excessive production of irregular and immature bones. ${ }^{[8]}$ Osteosarcomas can be differentiated based on the histological appearance. As per evidence, the most common subtypes of osteosarcomas include chondroblastic, fibroblastic, and osteoblastic variants. ${ }^{[9]}$ The diagnosis of osteosarcoma can be evident through radiological investigations such as computed tomography (CT) or MRI. The radiographic features may not be specific, wherein it could be osteoblastic, osteolytic, or mixed. ${ }^{[10]}$ In our case, we used a combination of PET-CT and histopathology to confirm the diagnosis.

There is no standard treatment for osteosarcoma of the skull which makes it a difficult and challenging case for oncologist. ${ }^{[10]}$ Surgical excision is considered as the standard treatment care which was followed in our case as well. ${ }^{[10]}$

Chemotherapy such as Adriamycin, cisplatin, methotrexate, ifosfamide, and epirubicin have been widely used for the treatment of osteosarcomas. ${ }^{[1]}$ In our case, the patient was treated with adjuvant Adriamycin and cisplatin. The diagnosis of osteosarcoma of the skull is based on the correlation of clinical, radiological, and histologic features as done in our case. ${ }^{[12]}$

\section{Conclusion}

Although osteosarcoma is a common aggressive tumor, its occurrence in the skull is usually rare. The diagnostic evaluation of osteosarcoma of the skull requires a multidisciplinary approach, i.e. clinical, radiological, and histopathological correlation. Due of our multidisciplinary approach, the patient was diagnosed and surgically treated at the earliest. The patient is currently doing well and due for her next follow-up. Although case reports are at the lowest in the evidence hierarchy, reporting unusual cases can help disseminate knowledge in the medical and scientific fraternity.

\section{Acknowledgement}

We would like to thank Mr Lyndon Fernandes for his medical writing assistance.

\section{References}

1. Misaghi A, Goldin A, Awad M, Kulidjian AA. Osteosarcoma: a comprehensive review. SICOT J. 2018;4:12.

2. Fernandes GL, Natal MRC, da Cruz CLP, Nascif RL, Tsuno NSG, Tsuno MY. Primary osteosarcoma of the cranial vault. Radiol Bras. 2017;50(4):263-265.

3. Taran SJ, Taran R, Malipatil NB. Pediatric Osteosarcoma: An Updated Review. Indian J Med Paediatr Oncol. 2017;38(1):33-43.

4. Arora RS, Eden TO, Kapoor G. Epidemiology of childhood cancer in India. Indian J Cancer. 2009 Oct-Dec;46(4):264-73.

5. Wu G, Liang Q, Liu Y. Primary osteosarcoma of frontal bone: A case report and review of literature. Medicine (Baltimore). 2017;96(51):e9392.

6. Salvati M, Ciappetta P, Raco A. Osteosarcomas of the skull. Clinical remarks on 19 cases. Cancer. 1993 Apr 1;71(7):2210-6.

7. Byrne J, Fears TR, Whitney C, Parry DM. Survival after retinoblastoma: long-term consequences and family history of cancer. Med Pediatr Oncol. 1995 Mar;24(3):160-5.

8. Oda D, Bavisotto LM, Schmidt RA, McNutt M, Bruckner JD, Conrad EU 3rd, Weymuller EA Jr. Head and neck osteosarcoma at the University of Washington. Head Neck. 1997 Sep;19(6):513-23.

9. Inwards CY, Unni KK. Classification and grading of bone sarcomas. Hematol Oncol Clin North Am. 1995 Jun;9(3):545-69.

10. Sofiene, B. , Asma, B. , Adnene, B. , Imed, B. , Jalel, K. , Lassaad, A. and Hafedh, J. (2014) A Case Report of Osteosarcoma of the Skull. Open Journal of Modern Neurosurgery, 4, 105-109.

11. Zhang Y, Yang J, Zhao N, et al. Progress in the chemotherapeutic treatment of osteosarcoma. Oncol Lett. 2018;16(5):6228-6237.

12. Cihan YB, Kaplan B, Deniz K, Dönmez H. Primary Calvarial Osteosarcoma: A Case Report. Journal of Neurological Sciences (Turkish), 2012; 29(1): 122-128.

Copyright: ( $\odot$ the author(s), publisher. Academia Anatomica International is an Official Publication of "Society for Health Care \& Research Development". It is an open-access article distributed under the terms of the Creative Commons Attribution Non-Commercial License, which permits unrestricted non-commercial use, distribution, and reproduction in any medium, provided the original work is properly cited.

How to cite this article: Adhav A, Bhalerao S, Nagarkar R, Roy S, Pawar S. Osteosarcoma of the Skull: An Unusual Presentation in the Left Parietal-Occipital Region. Acad. Anat. Int. 2019;5(1):10-11.

DOI: dx.doi.org/10.21276/aanat.2019.5.1.3

Source of Support: Nil, Conflict of Interest: None declared. 